\title{
New-Onset Diabetes Mellitus in Patients with Idiopathic Membranous Nephropathy Undergoing Tacrolimus and Low-Dose Corticosteroid Therapy
}

\author{
Lina Shao ${ }^{a-c}$ Juan Jin ${ }^{a-c}$ Binxian Ye ${ }^{a-c}$ Baihui Xu ${ }^{a-c}$ Yiwen Li ${ }^{a-c}$ \\ Jianguang Gong ${ }^{a-c}$ Jiong Zhang ${ }^{d}$ Maosheng Chen ${ }^{a-c}$ Qiang He $\mathrm{He}^{\mathrm{a}-\mathrm{c}}$ \\ a'Department of Nephrology, Zhejiang Provincial People's Hospital, Hangzhou, PR China; \\ ${ }^{b}$ People's Hospital of Hangzhou Medical College, Hangzhou, PR China; ${ }^{c}$ Chinese Medical \\ Nephrology Key Laboratory of Zhejiang Province, Hangzhou, PR China; ${ }^{d}$ Nephrology \\ National Clinical Research Center of Kidney Diseases, Jinling Hospital, Nanjing University \\ School of Medicine, Nanjing, PR China
}

\section{Keywords}

New-onset diabetes mellitus · Idiopathic membranous nephropathy · Tacrolimus ·

Corticosteroid · Age

\begin{abstract}
Background: Idiopathic membranous nephropathy (IMN) is the most common cause of nephrotic syndrome in adults. Although various studies have demonstrated the efficacy of tacrolimus combined with corticosteroids for treating IMN, both tacrolimus and corticosteroids have been shown to be diabetogenic, particularly following organ transplantation. Furthermore, the frequency and risk factors for new-onset diabetes mellitus (NODM) in IMN patients treated with tacrolimus plus low-dose corticosteroids remain unclear. Objectives: To evaluate the incidence of NODM in IMN patients undergoing tacrolimus plus low-dose corticosteroid therapy and to confirm the risk factors for NODM development. Methods: This retrospective study recruited 72 eligible patients with biopsy-proven IMN from our center, between September 2013 and June 2018. All subjects were treated with tacrolimus plus lowdose corticosteroids for a minimum of 3 months. The primary outcome was NODM development during the follow-up period. The secondary outcome was complete or partial remission. Patients were divided into 2 groups: patients with NODM (NODM group) and those
\end{abstract}




\section{Kidney \\ Blood Pressure \\ Research}

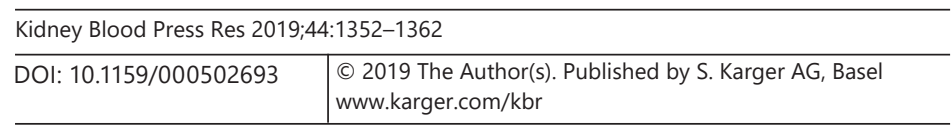

Shao et al.: NODM in IMN Patients Undergoing Tacrolimus and Low-Dose

Corticosteroid Therapy

without NODM (No-NODM group). Demographic and clinical data at baseline and follow-up were assessed. Results: During follow-up, 31 of the 72 patients developed NODM (43.0\%). The median time to occurrence was 3 months after treatment initiation. NODM patients were significantly older (median age 59 vs. 40 years) than No-NODM patients. Baseline fasting blood glucose levels were slightly higher in the NODM group; however, the difference was not significant $(p=0.07)$. Older age was an independent risk factor for NODM (OR 1.73 and $95 \% \mathrm{Cl} 1.20-2.47, p=0.003)$. Overall kidney remission rates were $80.6 \%$. There was no significant difference in remission rate between groups. There was a significant difference in development of pulmonary infection, which occurred in 7 NODM patients and only in 1 NoNODM patient $(p=0.018)$. IMN reoccurred in 5 NODM patients but only 1 No-NODM patient. Conclusions: Tacrolimus plus low-dose corticosteroid therapy was an efficient treatment for IMN; however, it was accompanied by increased NODM morbidity, which should be considered serious, due to the increased risk of life-threatening complications. Increasing age was a major risk factor for NODM in IMN patients treated with tacrolimus plus low-dose corticosteroid therapy.

(C) 2019 The Author(s)

Published by S. Karger AG, Basel

\section{Introduction}

Idiopathic membranous nephropathy (IMN) is the most common cause of nephrotic syndrome in adults. A previous study revealed that the frequency of membranous nephropathy (MN) increased over the study period, and long-term exposure to high levels of fine particles (PM 2.5) was associated with an increased risk of MN [1]. Although spontaneous remission occurs in approximately $30 \%$ of patients, many other patients require ongoing treatment with immunosuppressive drugs (ISDs). The Kidney Disease Improving Global Outcomes (KDIGO) guideline recommends a cyclical combination of cyclophosphamide and steroids as the first-line treatment for IMN [2]. Calcineurin inhibitors (CNIs) including tacrolimus and cyclosporine were recommended by the KDIGO as the only alternative regimens for initial therapy [2]. Several studies have demonstrated the efficacy of tacrolimus combined with corticosteroids as treatment for IMN [3-5]. A meta-analysis showed that efficacy of tacrolimus was comparable with that of cyclophosphamide for inducing renal remission of IMN patients within 1 year [6]. However, following application of tacrolimus for treatment of IMN, some patients developed hyperglycemia, indicating that these immunosuppressants can be diabetogenic. In general, corticosteroids and CNIs are considered to be the predominant factors for development of post-transplant diabetes mellitus [7].

The majority of current knowledge about the development of diabetes mellitus following the use of ISDs is derived from studies of steroid-induced diabetes in nonchronic kidney disease populations such as those with rheumatologic disease [8]. However, a better understanding of the incidence and outcomes of new-onset diabetes in patients with glomerulonephritis treated with ISD is lacking [9]. Furthermore, the incidence and risk factors for newonset diabetes mellitus (NODM) in IMN patients treated with tacrolimus plus low-dose corticosteroids remain unclear. The effectiveness of this therapy may be attenuated by NODM, which is an important risk factor for infection in IMN patients. In addition, treatment for NODM increases the cost; therefore, alternative treatments should be considered for patients with these risk factors.

This retrospective study evaluates the incidence of NODM in IMN patients who received tacrolimus plus low-dose corticosteroid therapy and identifies risk factors for the development of NODM. 
Shao et al.: NODM in IMN Patients Undergoing Tacrolimus and Low-Dose

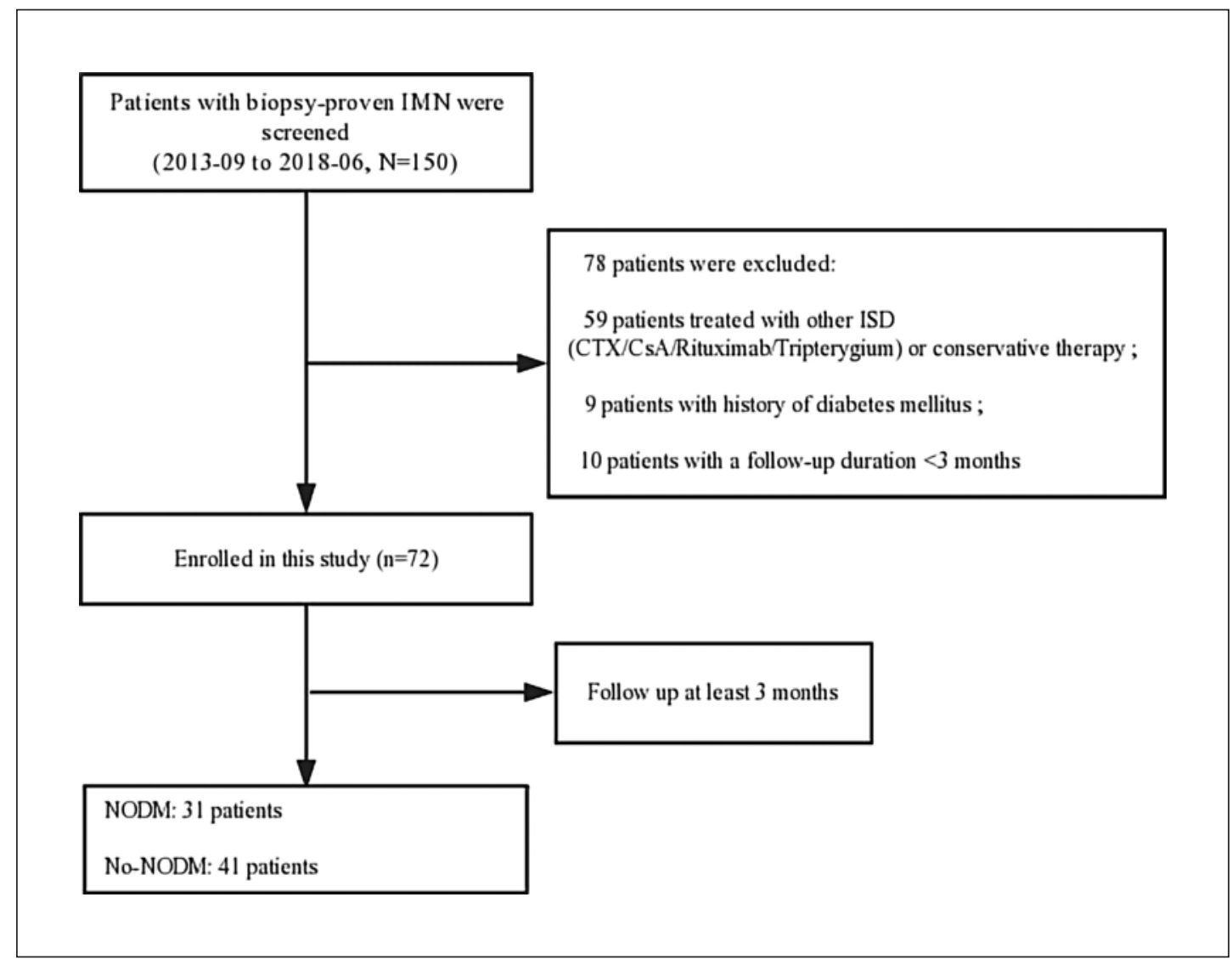

Fig. 1. Study screening process. Of the 150 patients who were screened for participation in the study, 78 patients were excluded, and 72 patients were enrolled in this study. IMN, idiopathic membranous nephropathy; ISD, immunosuppressive drug; NODM, new-onset diabetes mellitus.

\section{Materials and Methods}

\section{Patients}

A total of 150 patients from our center with biopsy-proven IMN were prospectively enrolled in this retrospective study between September 2013 and June 2018. Patients treated with other ISDs (cyclophosphamide, cyclosporine A, rituximab, or tripterygium) or conservative therapy $(n=59)$, those with a history of diabetes mellitus $(n=9)$ and those with a follow-up duration of $<3$ months $(n=10)$ were excluded from the study. A total of 72 patients with IMN were included in the study (Fig. 1). The study was approved by the Ethics Committee of Zhejiang Provincial People's Hospital. The need for informed consent was waived due to the retrospective study design.

\section{Treatment}

Oral prednisone was administered at an initial dose of $0.4 \mathrm{mg} / \mathrm{kg} /$ day and then reduced by $5 \mathrm{mg}$ every 4 weeks to $10 \mathrm{mg} /$ day for 6 months. Tacrolimus was administered at an initial dose of $0.05 \mathrm{mg} / \mathrm{kg} /$ day and adjusted according to target concentration to between 5 and $8 \mathrm{ng} / \mathrm{mL}$ [10]. Patients with hypertension received antihypertensive drugs to achieve a target level of $<130 / 80 \mathrm{~mm} \mathrm{Hg}$. Anticoagulant therapy was initiated in patients with severe hypoalbuminemia $(<20 \mathrm{~g} / \mathrm{L})[10]$. 


\section{Kidney \\ Blood Pressure \\ Research}

\begin{tabular}{l|l}
\hline Kidney Blood Press Res 2019;44:1352-1362 \\
\hline DOI: 10.1159/000502693 & $\begin{array}{l}\text { ○ 2019 The Author(s). Published by S. Karger AG, Basel } \\
\text { www.karger.com/kbr }\end{array}$ \\
\hline
\end{tabular}

Shao et al.: NODM in IMN Patients Undergoing Tacrolimus and Low-Dose

Corticosteroid Therapy

\section{Definitions}

Occurrence of NODM following treatment was defined according to the American Diabetes Association guidelines [11]. This requires a patient to experience symptoms of diabetes accompanied by a random plasma glucose concentration of $\geq 11.1 \mathrm{mmol} / \mathrm{L}$, a fasting plasma glucose concentration of $\geq 7.0 \mathrm{mmol} / \mathrm{L}$, a $2 \mathrm{~h}$ plasma glucose concentration of $\geq 11.1 \mathrm{mmol} / \mathrm{L}$ during an oral glucose tolerance test (OGTT), or plasma HbA1c of $\geq 6.5 \%$.

Patients were divided into 2 groups: patients with NODM (NODM group) and those without NODM (No-NODM group). The term remission includes both complete remission (CR) and partial remission (PR). CR was defined as proteinuria of $<0.3 \mathrm{~g} /$ day following initiation of treatment, confirmed by 2 measurements, obtained at least 1 week apart, with a normal serum albumin concentration and a normal serum creatinine. PR was defined as proteinuria of $<3.5 \mathrm{~g} /$ day and a $50 \%$ or greater reduction from peak values after treatment initiation, confirmed by 2 measurements, obtained at least 1 week apart.

\section{Data Collection}

Clinical, laboratory, and therapy data of the participants were recorded in the database of Department of Nephrology, Zhejiang Provincial People's Hospital, Hangzhou, China. Estimated glomerular filtration rate (eGFR) was calculated using the 2009 chronic kidney diseaseEPI creatinine equation [10].

\section{Statistical Analyses}

All statistical analyses were performed using SPSS 19.0 software (SPSS, Inc. Chicago, IL, USA). Demographic and clinical data are expressed as proportions or means \pm SD for normally distributed data or as medians with interquartile range for nonnormally distributed data. Differences in means between NODM and No-NODM groups were tested using a Student $t$ test, and data expressed as medians were tested using the nonparametric Mann-Whitney U test. Categorical variables between the 2 groups were compared using a chi-square test. Logistic regression analysis was performed for univariate analysis; then, the variable with a $p$ value of $<0.01$ in the univariate analysis was entered as the candidate variable in multivariate logistic regression model analysis, and an advanced logistic regression method was used for multivariate screening. The area under the receiver operating characteristic curve was used to evaluate the predictive value of the multivariate logistic regression model. All tests were bilateral, and a $p$ value of $<0.05$ was considered statistically significant in all analyses.

\section{Results}

\section{Patient Characteristics}

In total, 72 patients were enrolled in this study after screening (Fig. 1). There were 40 male and 32 female patients, and the median age was 51 years. The median follow-up period was 16.3 months (range 7.5-30.4 months). No patients had a family history of diabetes. Table 1 shows the comparison of the demographic and clinical variables between. Mean baseline proteinuria was 5.42 (range 3.78-9.1) g/day, albumin was $21.7 \pm 5.3 \mathrm{~g} / \mathrm{L}$, median serum creatine was 74.4 (range 66.1-88.2) $\mu \mathrm{mol} / \mathrm{L}$, and fasting blood glucose (FBG) was $4.9 \pm 0.57 \mathrm{mmol} / \mathrm{L}$. Only 40 patients were tested for blood PLA2R antibody (Ab) levels at baseline and $31(77.5 \%)$ yielded positive results. Renal biopsies indicated stages I-II lesions in 70 patients and stage III lesions in 2 patients.

\section{Morbidity of NODM}

NODM occurred in 31 patients between 2 weeks and 24 months after treatment (median time to occurrence: 3 months). The cumulative frequency of NODM increased over time, and 
Table 1. Baseline characteristics of the IMN patients receiving tacrolimus and low-dose corticosteroids treatments with or without NODM

\begin{tabular}{|c|c|c|c|c|}
\hline & $\begin{array}{l}\text { Total } \\
(n=72)\end{array}$ & $\begin{array}{l}\text { NODM } \\
(n=31)\end{array}$ & $\begin{array}{l}\text { No-NODM } \\
(n=41)\end{array}$ & $p$ value \\
\hline Follow-up time & $16.3(7.5-30.4)$ & $15.9(7.6-26.3)$ & $16.9(7.0-31.2)$ & 0.95 \\
\hline Age, years & $51(33-62)$ & $59(49-68)$ & $40(29-59)$ & 0.001 \\
\hline Male, $n(\%)$ & $40(55.6)$ & $17(54.8)$ & $23(56.1)$ & 0.92 \\
\hline Family history of hypertension & 2 & 0 & 2 & 0.50 \\
\hline Hypertension & 19 & 10 & 9 & 0.33 \\
\hline BMI, $\mathrm{kg} / \mathrm{m}^{2}$ & $24.4 \pm 3.4$ & $24.7 \pm 3.2$ & $24.1 \pm 3.6$ & 0.43 \\
\hline Albumin, g/L & $21.7 \pm 5.3$ & $21.3 \pm 4.4$ & $22 \pm 5.9$ & 0.60 \\
\hline FBG, mmol/L & $4.9 \pm 0.57$ & $5.07 \pm 0.49$ & $4.83 \pm 0.61$ & 0.07 \\
\hline Scr, $\mu \mathrm{mol} / \mathrm{L}$ & $74.4(66.1-88.2)$ & $71.4(66.1-79.8)$ & $76.1(65.4-92.0)$ & 0.27 \\
\hline $\mathrm{eGFR}, \mathrm{mL} / \mathrm{min} \times 1.73 \mathrm{~m}^{2}$ & $92.6 \pm 25.8$ & $90.7 \pm 21.2$ & $94.1 \pm 29$ & 0.57 \\
\hline Uric acid, $\mu \mathrm{mol} / \mathrm{L}$ & $372 \pm 91$ & $361 \pm 93$ & $380 \pm 90$ & 0.38 \\
\hline $\mathrm{TG}, \mathrm{mmol} / \mathrm{L}$ & $2.1(1.5-2.9)$ & $2.1(1.7-2.7)$ & $2.0(1.47-3.0)$ & 0.40 \\
\hline LDL-c, mmol/L & $5.4 \pm 2.7$ & $4.93 \pm 3.0$ & $5.74 \pm 2.3$ & 0.20 \\
\hline $24 \mathrm{~h}$ urine protein, $\mathrm{g}$ & $5.42(3.78-9.1)$ & $6.1(4.4-10.7)$ & $4.81(3.1-9.1)$ & 0.29 \\
\hline PLA2R antibody positive ${ }^{\mathrm{a}}, n(\%)$ & $31(77.5)$ & 15 & 16 & 0.12 \\
\hline PLA2R antibody negative ${ }^{\mathrm{a}}, n(\%)$ & $9(22.5)$ & 4 & 5 & \\
\hline GC history, $n$ & 6 & 4 & 2 & 0.39 \\
\hline \multicolumn{5}{|l|}{ Pathological stage, $n(\%)$} \\
\hline Stage I & $33(45.8)$ & $11(35.5)$ & $22(53.7)$ & 0.31 \\
\hline Stage II & $37(51.4)$ & $18(61.3)$ & $19(43.9)$ & \\
\hline Stage III & $2(2.8)$ & $1(3.2)$ & $1(2.4)$ & \\
\hline
\end{tabular}

FBG, fasting blood-glucose; Scr, serum creatinine; BMI, body mass index; TG, triglyceride; GC history, history of glucocorticoid use before come to our hospital for treatment; LDL-C, low-density lipoprotein cholesterol; IMN, idiopathic membranous nephropathy; NODM, new-onset diabetes mellitus. ${ }^{\text {a }}$ Only 40 patients were monitored blood level of PLA2R Ab at the base line. The other patients had no those PLA2R antibody data because we haven't implemented this testing technology yet when they were diagnosed.

Fig. 2. The cumulative frequency of NODM increased over time during follow-up time.

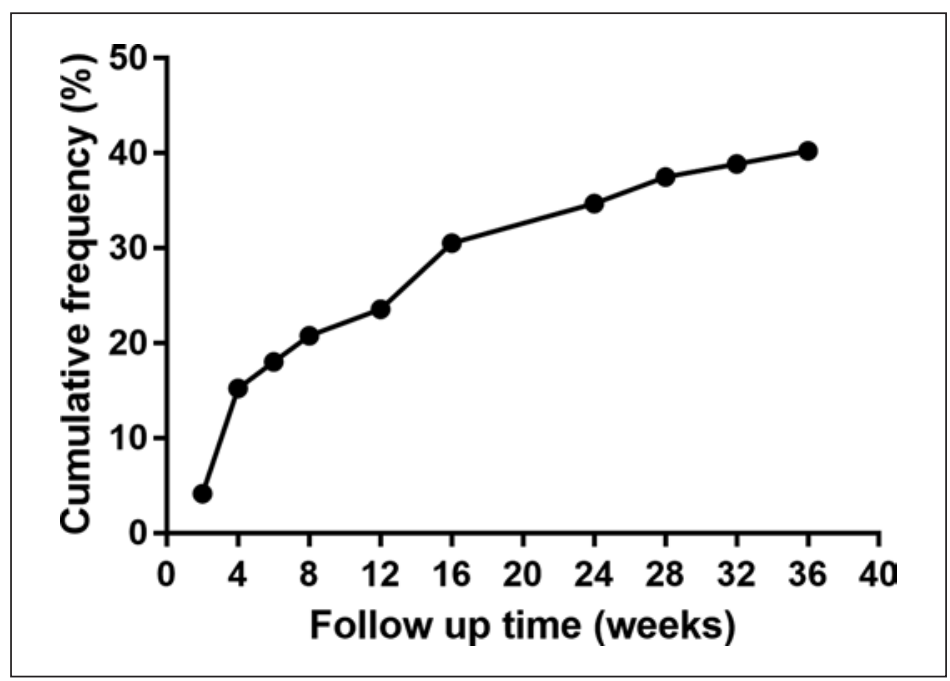

the 2-, 4-, 8-, 12-, 16-, 24-, and 36-month frequency rates were 4.2, 15.3, 20.8, 23.6, 30.6, 34.7, and $40.28 \%$, respectively (Fig. 2). The median time to occurrence was 3 months after treatment initiation. The median HbA1c was 7.85\%. The median FBG level was $9.37 \mathrm{mmol} / \mathrm{L}$ (Table 2). 
Table 2. Comparison of outcomes and complications

\begin{tabular}{|c|c|c|c|c|}
\hline & Total & NODM & No-NODM & $p$ value \\
\hline Follow-up time & $16.3(7.5-30.4)$ & $15.9(7.6-26.3)$ & $16.9(7.0-31.2)$ & 0.95 \\
\hline $\mathrm{HbA1c}, \%^{\mathrm{a}}$ & - & $7.85(6.75-9.83)$ & - & \\
\hline $\mathrm{FBG}, \mathrm{mmol} / \mathrm{L}$ & $5.93(4.71-8.7)$ & $9.37(7.63-12.7)$ & $4.73(4.43-5.1)$ & 0.000 \\
\hline Time after ISD therapy, month & - & $3(1-6)$ & - & \\
\hline \multicolumn{5}{|l|}{ Life outcomes } \\
\hline Live & 71 & 30 & 41 & 0.43 \\
\hline Died & 1 & 1 & 0 & \\
\hline \multicolumn{5}{|l|}{ Kidney outcomes, $n(\%)$} \\
\hline $\mathrm{CR}$ & $40(55.6)$ & $20(64.5)$ & $20(48.8)$ & - \\
\hline PR & $18(25)$ & $8(25.8)$ & $10(24.4)$ & \\
\hline $\mathrm{CR}+\mathrm{PR}$ & $58(80.6)$ & $28(90.3)$ & $30(73.2)$ & 0.069 \\
\hline NR & $14(19.4)$ & $3(9.7)$ & $11(26.8)$ & \\
\hline Remission time, month & $6.75(4.0-11.5)$ & $7.25(5.8-11.7)$ & $5.1(3.1-10.64)$ & 0.22 \\
\hline eGFR (remission), $\mathrm{mL} / \mathrm{min}$ & $91.3 \pm 23.1$ & $86.7 \pm 23.86$ & $95.6 \pm 22.2$ & 0.15 \\
\hline Fk506, ng/mL & $5.0 \pm 2.42$ & $5.61 \pm 2.51$ & $4.61 \pm 2.33$ & 0.17 \\
\hline Recurrence, $n(\%)$ & $6(8)$ & $5(16.1)$ & $1(2.4)$ & 0.08 \\
\hline Severe lung infection, $n(\%)$ & $8(11)$ & $7(22.6)$ & $1(2.4)$ & 0.02 \\
\hline Severe lung infection occur time & $4(2.25-6)$ & $4(3-6)$ & 2 & - \\
\hline
\end{tabular}

Fk506 (ng/mL): The plasma concentration of tacrolimus; eGFR ( $\mathrm{mL} / \mathrm{min} / 1.73 \mathrm{~m}^{2}$ ), calculated by the CKD-EPI formula for remission patients. FBG, fasting blood-glucose; CR, complete remission; PR, partial remission; NR, no remission; eGFR, estimate glomerular filtration rate. ${ }^{a}$ The blood level of HbA1c was not conducted in the No-NODM group because their normal fasting blood-glucose level.

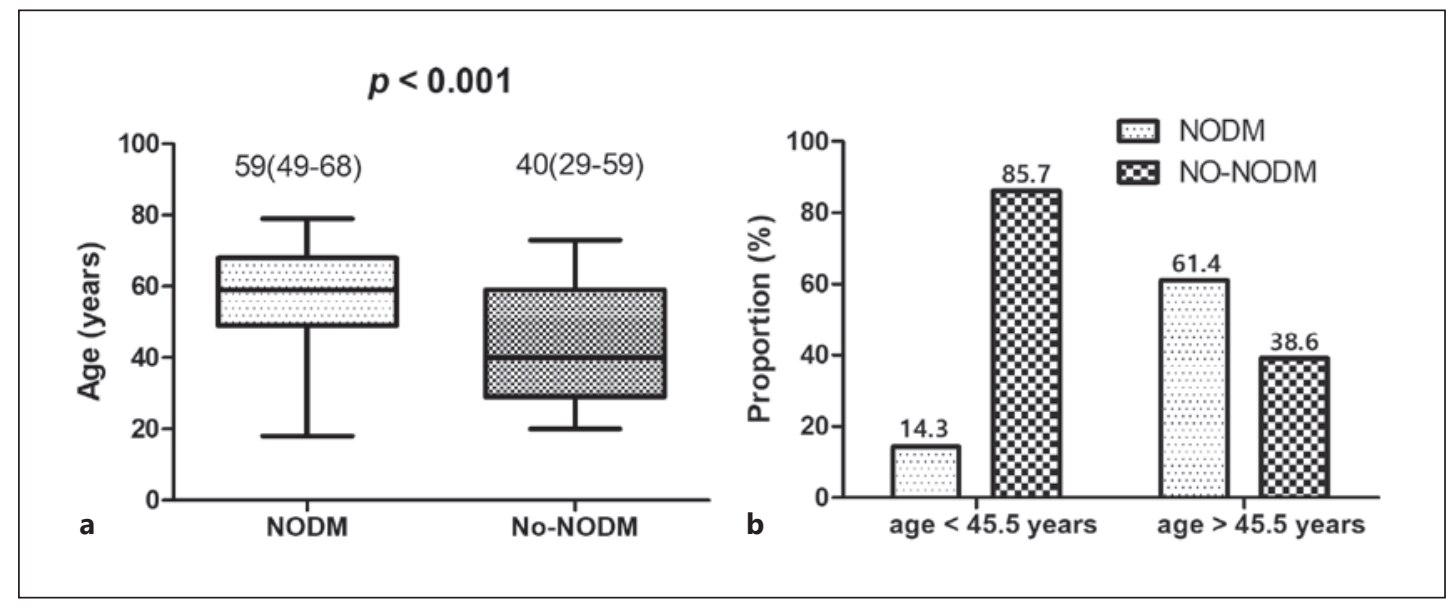

Fig. 3. a Age distribution in 2 groups; NODM. b The proportion of NODM in 2 different age groups. NODM, new-onset diabetes mellitus.

\section{Characteristics of Patients with NODM}

Patients with NODM were significantly older than those without NODM (median age 59 vs. 40 years, $p=0.001$; Fig. 3a). Baseline FBG was higher in the NODM group, but the difference was not significant (median level 5.07 vs. 4.83, $p=0.07$ ). Family history of hypertension, personal history of hypertension, body mass index, serum albumin level, serum creatinine, eGFR, low-density lipoprotein cholesterol, triglycerides, 24-h urinary protein, hyperuricemia, PLA2R antibody positivity, pathological stage, and history of glucocorticoid use did not differ between NODM and No-NODM groups. 


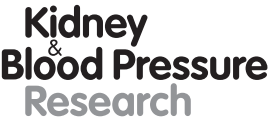

Research

\begin{tabular}{l|l}
\hline Kidney Blood Press Res 2019;44:1352-1362 \\
\hline DOI: 10.1159/000502693 & $\begin{array}{l}\text { @ 2019 The Author(s). Published by S. Karger AG, Basel } \\
\text { www.karger.com/kbr }\end{array}$ \\
\hline
\end{tabular}

Shao et al.: NODM in IMN Patients Undergoing Tacrolimus and Low-Dose Corticosteroid Therapy

Table 3. Predictive factors of NODM after initiation of tacrolimus and low-dose corticosteroids therapy

\begin{tabular}{|c|c|c|c|c|}
\hline & \multicolumn{2}{|l|}{ Univariate } & \multicolumn{2}{|l|}{ Multivariate } \\
\hline & HR and $95 \% \mathrm{CI}$ & $p$ value & HR and 95\% CI & $p$ value \\
\hline Age at baseline (per 10 years) & $1.75(1.24-1.48)$ & 0.002 & $1.73(1.20-2.47)$ & 0.003 \\
\hline Male & $1.05(0.41-2.69)$ & 0.92 & & \\
\hline Hypertension & $1.69(0.59-4.87)$ & 0.33 & & \\
\hline GC history & $2.89(0.49-16.9)$ & 0.24 & $2.5(0.32-19.4)$ & 0.381 \\
\hline Overweight & $1.77(0.69-4.54)$ & 0.24 & $1.67(0.52-4.74)$ & 0.34 \\
\hline Albumin, g/L & $0.98(0.89-1.07)$ & 0.60 & & \\
\hline $\mathrm{FBG}, \mathrm{mmol} / \mathrm{L}$ & $2.18(0.92-5.15)$ & 0.08 & $2.07(0.79-5.43)$ & 0.14 \\
\hline eGFR & $1.21(0.58-2.51)$ & 0.61 & & \\
\hline Hyperuricemia & $0.46(0.22-1.39)$ & 0.17 & & \\
\hline $\mathrm{TG}, \mathrm{mmol} / \mathrm{L}$ & $1.19(0.88-1.61)$ & 0.26 & & \\
\hline LDL-C, mmol/L & $0.88(0.72-1.07)$ & 0.20 & & \\
\hline 24 h-urine protein, $g$ & $1.05(0.95-1.17)$ & 0.34 & & \\
\hline
\end{tabular}

GC history, history of glucocorticoid use. Overweight: BMI $\geq 24$. FBG. eGFR (mL/min $\left./ 1.73 \mathrm{~m}^{2}\right)$, calculated by the CKD-EPI formula for remission patients. Hyperuricemia: male $\geq 420 \mu \mathrm{mol} / \mathrm{L}$ and female $\geq 380 \mu \mathrm{mol} / \mathrm{L}$. TG, triglyceride; LDL-C, low-density lipoprotein cholesterol; NODM, new-onset diabetes mellitus; FBG, fasting blood-glucose. eGFR, estimate glomerular filtration rate; HR, hazard ratio.

\section{Risk Factors for NODM}

Univariate logistic regression indicated that age and baseline FBG level were risk factors for NODM (Table 3). Since the relationship between being overweight (body mass index $\geq 24$ ) and insulin resistance is clear, being overweight was included in the further analysis. We add history of glucocorticoid use in multivariate analysis because of its side effect of raising blood sugar. A multivariate logistic regression model was established with NODM as a dependent variable and age, history of glucocorticoid use, being overweight, and FBG level as independent variables.

Age was identified as the only independent risk factor for NODM in patients with IMN following tacrolimus plus low-dose corticosteroid therapy (OR 1.73, 95\% CI 1.20-2.47, $p=0.003$ ). The risk of developing NODM increased 1.73 times for every 10 years of age (Table 3). The predictive value of the multivariate logistic regression model was 0.723 . The cutoff value of age for developing NODM was 45.5 years, based on the Youden index. The area under curve was $0.73(p=0.001)$. Only $14.3 \%$ patients who were younger than 45.5 years developed NODM, and $61.4 \%$ patients who were older than 45.5 years developed NODM, suggesting that tacrolimus plus low-dose corticosteroid therapy should be avoided in IMN patients older than 45.5 years, taking into consideration the increased risk of developing NODM (Fig. 3b).

\section{Outcomes and Complications of NODM}

The median follow-up time was 16.3 months (range 7.5-30.4). One patient in the NODM group died due to severe pneumonia. The median FBG level was 9.37 and $4.73 \mathrm{mmol} / \mathrm{L} \mathrm{in}$ NODM and No-NODM groups and the difference was significant. The overall kidney remission of patients was $80.6 \%$; CR was 64.5 and $48.8 \%$ in NODM and No-NODM groups, respectively. PR was 25.8 and $24.4 \%$ in NODM and No-NODM groups, respectively. The total remission rate was not significantly different between the groups $(p=0.069)$. The median remission time was 7.3 and 5.1 months in NODM and No-NODM groups; however, the difference was not significant. The eGFR of remission patients was $86.7 \pm 23.86$ and $95.6 \pm 22.2 \mathrm{~mL} / \mathrm{min}$ in NODM and No-NODM groups, respectively, however, no significant difference was revealed. Recur- 


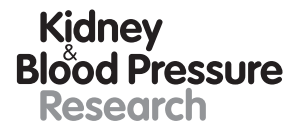

Research \begin{tabular}{l|l}
\hline Kidney Blood Press Res 2019;44:1352-1362 \\
\hline DOI: 10.1159/000502693 & $\begin{array}{l}\text { @ 2019 The Author(s). Published by S. Karger AG, Basel } \\
\text { www.karger.com/kbr }\end{array}$ \\
\hline
\end{tabular}

Shao et al.: NODM in IMN Patients Undergoing Tacrolimus and Low-Dose

Corticosteroid Therapy

rence reoccurred in 5 NODM patients but only one No-NODM patient; however, this was not significantly different between groups, respectively. There was a significant difference in development of pulmonary infection, which occurred in 7 NODM patients and only 1 No-NODM patient $(p=0.018)$.

\section{Discussion/Conclusion}

The morbidity of NODM in patients with IMN undergoing tacrolimus plus low-dose corticosteroid therapy was high (40.28\%), and the cumulative frequency increased over time. The median age in this study was 51 years old, and the range between the first quartile and the third quartile was 33-62 years. This age range was both representative of and similar to that reported in previous studies of MN $[12,13]$. Older age was the only risk factor for NODM, suggesting that older age should be carefully considered when developing treatment strategies for IMN patients, in order to avoid NODM. An age of $<45.5$ years was associated with a lower incidence of NODM (14.3\%), which is consistent with the results of another recently published study that focused on IgA nephropathy treated with methylprednisolone pulse therapy [9].

Recent studies demonstrated that greater proteinuria and methylprednisolone use were significantly associated with NODM, especially in patients older than 50 years. Although these studies included several kinds of glomerulonephritis with various ISD treatments $[14,15]$, older age was the major risk factor identified for NODM in these studies. These results suggest that IMN patients who are older than 45.5 years might not be suitable for tacrolimus plus low-dose corticosteroid therapy and should consider alternative ISD treatments.

A meta-analysis indicated that glucocorticoid-induced hyperglycemia occurs fairly frequently; the rate at which patients developed glucocorticoid-induced hyperglycemia or diabetes was $32.3 \%(p=0.003)$ and $18.6 \%(p=0.002)$, respectively [8]. The study patients showed a higher incidence of NODM, partly due to superimposed effects of low-dose corticosteroid and tacrolimus. The possible mechanism of diabetogenic effects of tacrolimus was considered to be decreased insulin secretion due to islet cell necrosis and increased insulin resistance, observed in a rat model of tacrolimus-induced diabetes [16]. Glucose tolerance progressively declines with age [17], which might be caused by the shortened telomeres of $\beta$ and $\alpha$ cells [18] and age-related changes to DNA methylation in human islet cells [19]. These two effects may explain the main study finding that older age was the only risk factor for the development of NODM. A study has revealed that liver transplant patients with NODM may benefit from conversion to cyclosporine from tacrolimus, as this can lead to improved glucose metabolism [20]. Since cyclosporine is the second-line ISD for IMN according to KDIGO [2], it could be a suitable alternative for these patients who develop NODM. However, further confirmation of these findings in a prospective, randomized comparative study is required.

Tacrolimus plus low-dose corticosteroid therapy was an efficient treatment for IMN. A systematic review and meta-analysis of 21 clinical trials showed that CNIs are promising alternatives to CTX for IMN patients and tacrolimus shows more lasting therapeutic effects and fewer side effects [21]. The present study showed that the overall remission was $80.6 \%$, which is similar to that reported in published studies [22, 23]. Rituximab was considered as another effective therapy for IMN [24]. However, IMN patients are unlikely to be prescribed rituximab in our developing country (China), as it is not covered by medical insurance for IMN treatment now. Furthermore, a multicenter prospective study indicated that low-dose rituximab is poorly effective in patients with IMN, which might 
Shao et al.: NODM in IMN Patients Undergoing Tacrolimus and Low-Dose

Corticosteroid Therapy

explain the higher costs [25]. Several clinical studies have demonstrated that rituximab is more effective for refractory MN [26], indicating that tacrolimus plus low-dose corticosteroid therapy is efficient and could be a first-line choice for MN patients who cannot afford the high cost of rituximab.

The NODM group had a higher incidence rate of pulmonary infection than the No-NODM group following tacrolimus plus low-dose corticosteroid therapy. One patient died due to severe pulmonary infection and extremely high glucose levels. The overall time to occurrence of pulmonary infection for NODM patients was 4 months (range 3-6). Although the remission rate was similar in both groups, this result suggests that development of NODM leads to worse outcomes, and it should be tightly controlled.

There are several limitations in this retrospective study. First, an OGTT was not performed for all patients before treatment initiation; therefore, patients with impaired OGTT but normal FBG levels may have been included. Therefore, an OGTT test should be performed before initiation of ISD in future studies. Second, the study sample size was small. Further studies are required to assess the incidence and outcomes of NODM in MN with ISD treatment.

Tacrolimus plus low-dose corticosteroid therapy was efficient for treatment of IMN but was accompanied by a high frequency of NODM. Frequency of NODM should be considered serious, due to the increased risk of life-threatening complications. Increasing age was a major risk factor for NODM in IMN patients undergoing tacrolimus plus low-dose corticosteroid therapy. Older IMN patients might not be suitable for tacrolimus plus low-dose glucocorticoid therapy, and these patients should consider alternative ISD treatments.

\section{Acknowledgments}

We would like to thank our colleagues at Zhejiang Provincial People's Hospital for their valuable contributions to this work.

\section{Statement of Ethics}

The research was approved by the Ethics Committee of Zhejiang Provincial People's Hospital. The need for informed consent was waived because of the retrospective study design.

\section{Disclosure Statement}

The authors declare that they have no competing interests. The authors have no conflicts of interest to declare.

\section{Funding Sources}

The authors acknowledge support from the Medical and Health Technology Program of Zhejiang Province (2019KY312), Zhejiang Traditional Chinese Medicine Science and Technology Program (2015ZZ002), and the Natural Sciences Fund of Zhejiang Province (LY19H05003). The funders had no role in study design, collection, analysis, interpretation of data, writing the report, or in the decision to submit the report for publication. 


\section{Kidney \\ Blood Pressure \\ Research}

\begin{tabular}{l|l}
\hline Kidney Blood Press Res 2019;44:1352-1362 \\
\hline DOI: 10.1159/000502693 & $\begin{array}{l}\text { @ 2019 The Author(s). Published by S. Karger AG, Basel } \\
\text { www.karger.com/kbr }\end{array}$ \\
\hline
\end{tabular}

Shao et al.: NODM in IMN Patients Undergoing Tacrolimus and Low-Dose

Corticosteroid Therapy

\section{Author Contributions}

L.S., M.C., and Q.H.: conceptualization. L.S., J.J., J.G., B.Y., and B.X.: data curation. L.S.: formal analysis. L.S., B.X., and Y.L.: investigation. L.S., Q.H., and M.C.: methodology. M.C. and Q.H.: supervision. L.S.: writing the original draft. L.S., M.C., and Q.H.: writing, review, and editing. All authors read and approved the final manuscript.

\section{References}

1 Xu X, Wang G, Chen N, Lu T, Nie S, Xu G, et al. Long-Term Exposure to Air Pollution and Increased Risk of Membranous Nephropathy in China. J Am Soc Nephrol. 2016 Dec;27(12):3739-46.

2 Radhakrishnan J, Cattran DC. The KDIGO practice guideline on glomerulonephritis: reading between the (guide)lines-application to the individual patient. Kidney Int. 2012 Oct;82(8):840-56.

3 Ramachandran R, Hn HK, Kumar V, Nada R, Yadav AK, Goyal A, et al. Tacrolimus combined with corticosteroids versus Modified Ponticelli regimen in treatment of idiopathic membranous nephropathy: randomized control trial. Nephrology (Carlton). 2016 Feb;21(2):139-46.

$4 \mathrm{Xu} \mathrm{J}$, Zhang W, Xu Y, Shen P, Ren H, Wang W, et al. Tacrolimus combined with corticosteroids in idiopathic membranous nephropathy: a randomized, prospective, controlled trial. Contrib Nephrol. 2013;181:15262.

5 Chen M, Li H, Li XY, Lu FM, Ni ZH, Xu FF, et al.; Chinese Nephropathy Membranous Study Group. Tacrolimus combined with corticosteroids in treatment of nephrotic idiopathic membranous nephropathy: a multicenter randomized controlled trial. Am J Med Sci. 2010 Mar;339(3):233-8.

6 Zhu LB, Liu LL, Yao L, Wang LN. Efficacy and Safety of Tacrolimus Versus Cyclophosphamide for Primary Membranous Nephropathy: A Meta-Analysis. Drugs. 2017 Feb;77(2):187-99.

7 Penfornis A, Kury-Paulin S. Immunosuppressive drug-induced diabetes. Diabetes Metab. 2006 Dec;32(5 Pt 2):539-46.

8 Liu XX, Zhu XM, Miao Q, Ye HY, Zhang ZY, Li YM. Hyperglycemia induced by glucocorticoids in nondiabetic patients: a meta-analysis. Ann Nutr Metab. 2014;65(4):324-32.

9 Miyawaki Y, Katsuyama T, Sada KE, Hiramatsu S, Ohashi K, Morishita M, et al. A retrospective observational study of glucocorticoid-induced diabetes mellitus with IgA nephropathy treated with tonsillectomy plus methylprednisolone pulse therapy. PLoS One. 2017 May;12(5):e0178018.

10 Andrassy KM. Comments on 'KDIGO 2012 Clinical Practice Guideline for the Evaluation and Management of Chronic Kidney Disease'. Kidney Int. 2013 Sep;84(3):622-3.

11 Marathe PH, Gao HX, Close KL. American Diabetes Association Standards of Medical Care in Diabetes 2017. J Diabetes. 2017 Apr;9(4):320-4.

12 Cattran DC, Kim ED, Reich H, Hladunewich M, Kim SJ; Toronto Glomerulonephritis Registry group. Membranous Nephropathy: Quantifying Remission Duration on Outcome. J Am Soc Nephrol. 2017 Mar; 28(3):995-1003.

13 Stanescu HC, Arcos-Burgos M, Medlar A, Bockenhauer D, Kottgen A, Dragomirescu L, et al. Risk HLA-DQA1 and PLA(2)R1 alleles in idiopathic membranous nephropathy. N Engl J Med. 2011 Feb;364(7):616-26.

14 Lim CC, Wong MW, Koh HL, Chin YM, Mok IY, Choo JC. New-onset Diabetes Mellitus among Patients with Glomerular diseases. Intern Med J. 2019 Jan;49(1):101-8.

15 Lim CC, Wong MWY, Koh HL, Mok IYJ, Chin YM, Choo JCJ. Methylprednisolone and greater proteinuria predispose older adults with glomerulonephritis to new onset diabetes mellitus. Diabetes Metab. 2018, Epub ahead of print.

16 Niu YJ, Shen ZY, Xu C, Li CF, Liu XJ, Teng YQ, et al. Establishment of tacrolimus-induced diabetes in rat model and assessment of clinical treatments for post-transplant diabetes mellitus in liver transplant recipients. Clin Lab. 2013;59(7-8):869-74.

17 Chang AM, Halter JB. Aging and insulin secretion. Am J Physiol Endocrinol Metab. 2003 Jan; 284(1):E7-12.

18 Tamura Y, Izumiyama-Shimomura N, Kimbara Y, Nakamura K, Ishikawa N, Aida J, et al. Telomere attrition in beta and alpha cells with age. Age (Dordr). 2016 Jun;38(3):61.

19 Bacos K, Gillberg L, Volkov P, Olsson AH, Hansen T, Pedersen O, et al. Blood-based biomarkers of age-associated epigenetic changes in human islets associate with insulin secretion and diabetes. Nat Commun. 2016 Mar;7(1):11089.

20 Lorho R, Hardwigsen J, Dumortier J, Pageaux GP, Durand F, Bizollon T, et al. Regression of new-onset diabetes mellitus after conversion from tacrolimus to cyclosporine in liver transplant patients: results of a pilot study. Clin Res Hepatol Gastroenterol. 2011 Jun;35(6-7):482-8.

21 Qiu TT, Zhang C, Zhao HW, Zhou JW. Calcineurin inhibitors versus cyclophosphamide for idiopathic membranous nephropathy: A systematic review and meta-analysis of 21 clinical trials. Autoimmun Rev. 2017 Feb;16(2):136-45.

22 Huh H, Lee H, Lee JP, Kim DK, Oh S, Oh YK, et al. Factors affecting the long-term outcomes of idiopathic membranous nephropathy. BMC Nephrol. 2017 Mar;18(1):104. 
23 Qin HZ, Liu L, Liang SS, Shi JS, Zheng CX, Hou Q, et al. Evaluating tacrolimus treatment in idiopathic membranous nephropathy in a cohort of 408 patients. BMC Nephrol. 2017 Jan;18(1):2.

24 Dahan K, Debiec H, Plaisier E, Cachanado M, Rousseau A, Wakselman L, et al.; GEMRITUX Study Group. Rituximab for Severe Membranous Nephropathy: A 6-Month Trial with Extended Follow-Up. J Am Soc Nephrol. 2017 Jan;28(1):348-58.

25 Moroni G, Depetri F, Del Vecchio L, Gallelli B, Raffiotta F, Giglio E, et al. Low-dose rituximab is poorly effective in patients with primary membranous nephropathy. Nephrol Dial Transplant. 2017 Oct;32(10): 1691-6.

26 Wang X, Cui Z, Zhang YM, Qu Z, Wang F, Meng LQ, etal. Rituximab for non-responsive idiopathic membranous nephropathy in a Chinese cohort. Nephrol Dial Transplant. 2018 Sep;33(9):1558-63. 